\title{
Claus Grimm
}

\section{Histoire du cadre : un panorama}

On n'a guère décrit jusqu'ici que des cadres de caractère artisanal exécutés d'une manière extrêmement riche. Nous tentons de dégager les lignes de développement propres aux principaux types de cadres européens.

L'encadrement des tableaux appartient, comme un complément nécessaire, à l'histoire du retable et à celle du tableau mobile, indépendant, qui est issu du retable. Si on examine l'histoire du retable dans son ensemble, notre histoire du cadre commence au XIII siècle ; mais si on considère seulement le tableau indépendant, elle commence à la fin du Xve siècle. Partant du fait qu'avec la photographie, les courants abstraits en peinture, les moyens modernes de reproduction et les manières de créer propres à la seconde moitié du $\mathrm{Xx}^{\mathrm{e}}$ siècle qui abandonnent le champ d'illusions du tableau, les possibilités du tableau sont " épuisées », on peut fixer au temps présent la fin provisoire du tableau et du cadre.

Le « cadre » en tant que forme artistique est un exemple unique, si on l'observe dans son extension temporelle, et il l'est également quand on se réfère aux différentes cultures. Il suffit de penser aux représentations extrêmement raffinées de la peinture et du dessin chinois ou japonais qui ne se sont jamais enfermés dans un champ visuel solidement charpenté.

Werner Ehlich a avancé des arguments en faveur

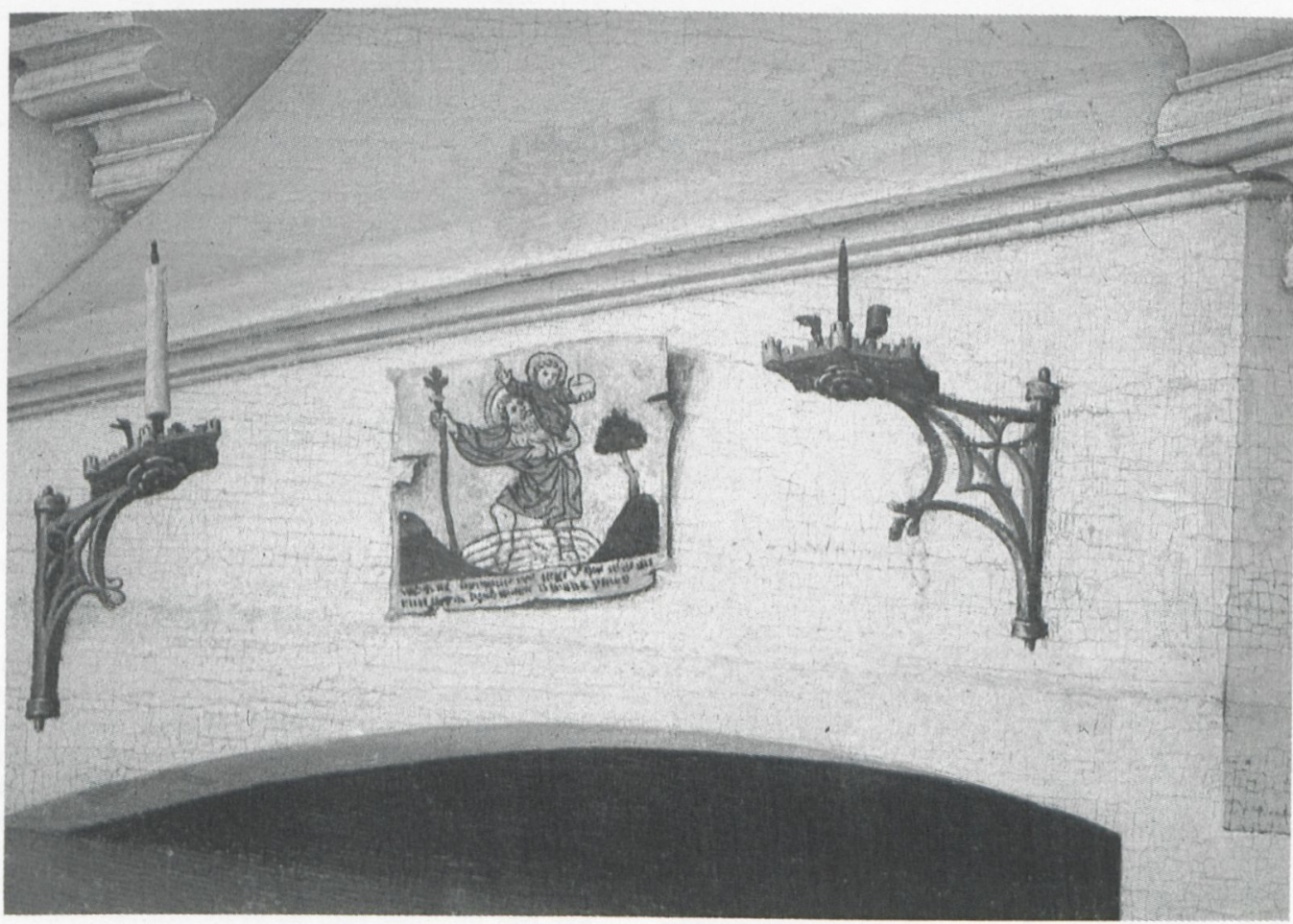

1. "Images " dans un intérieur du XVe siècle: détail de L'Annonciation du Maître de Flémalle, vers 1425, Bruxelles, Musées Royaux des Beaux-Arts. 
de l'existence dans l'Antiquité de formes simples, annonciatrices du cadre indépendant ${ }^{1}$. On peut cependant douter que celles-ci aient eu une signification artistique et esthétique comparable à celle des cadres qui sont venus par la suite. Mais on ne saurait sousestimer la relation à l'époque moderne du corps du retable (Altargehäuse) avec les éléments de l'architecture antique. L'ornement architecturé emprunte directement ses formes aux modèles antiques, en particulier le profil et les moulures. La nouveauté du " cadre " réside précisément en ce qu'il dispose des éléments de l'architecture, et en premier lieu de l'antique pour créer un objet d'ameublement, un " meuble ».

Il ressort clairement des quelques exemples cités que la succession historique des différents types de cadres ne peut pas être répartie entre de simples groupes stylistiques. Car cet « art " n'est pas tout uniment resté le même en ne revêtant que des habits de style différents, ce sont au contraire les conceptions fondamentales des cadres qui se sont modifiées. Si on veut contourner les problèmes non encore résolus de l'« histoire de l'art ", il ne faut pas perdre de vue l'autotransformation de l'objet au cours de son évolution historique.

Les historiens des sciences et les sociologues ont tenté d'écrire l'histoire de l'évolution comme une histoire des institutions, à savoir, par quels types de savants et d'institutions scientifiques sont portés les différents types de sciences? Si on adopte ce point de départ pour l'histoire du cadre, on ne remonte guère plus haut que la fin du XVIII siècle, époque où les créations les plus dispendieuses sont déjà du passé. Dans l'Encyclopédie de Diderot et d'Alembert, de $1751^{2}$, on voit, par exemple, des gravures représentant un atelier où on pratique exclusivement les activités propres à la fabrication des cadres : fabrication et assemblage des baguettes, sculpture et pose des moulures profilées -

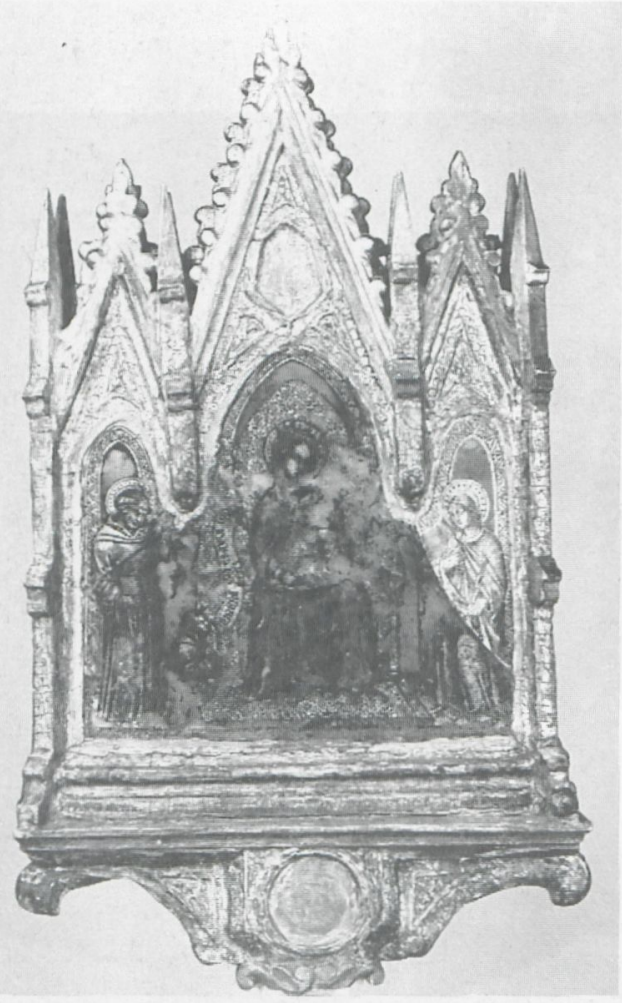

2. Cadre aux formes architectoniques d'un panneau de bois peint et fixé sur une hampe pour être porté dans les processions, peint aux deux faces par Francesco di Vanuccio, signé et daté 1370, Berlin-Dahlem, Gemäldegalerie. déjà préfabriquées et vendues au mètre - , et dorure. A la même époque, il était devenu usuel de signer les cadres - du moins dans les ateliers spécialisés et dans les grands ateliers de meubles des cours et des capitales. Mais, le traitant consciemment comme une réalisation artistique, il arrivait qu'on lui donnât un caractère de pauvreté. Le cadre du portrait de Diderot par Greuze emprunte volontairement des formes historiques ; du point de vue de l'effet esthétique, il a l'air, comparé aux œuvres - anonymes - de l'époque Louis XV, d'une robe en toile de jute à côté d'un brocart d'or.

On pourrait objecter que l'institution était tardive mais, en fait, déjà auparavant des créateurs de premier plan avaient déjà conscience de leur valeur. N'y a-t-il pas eu le maître Doomer dont Rembrandt a fait le portrait ${ }^{3}$, et le père de Jacob Ruisdael ${ }^{4}$, spécialisé dans la fabrication des cadres? Qu'en est-il des fabricants de cadres aussi éminents qu'Andrea Marchesi da Formigine, contemporain de Raphaël, que Jacopo da Faenza, qui a encadré le triptyque de Bellini à l'église des Frari, ou qu'Antonio Barile, auteur du cadre de la Sainte Famille de Michel-Ange au musée des Offices? Pour la réponse à ces questions, nous renvoyons aux informations réunies, en particulier, par Lerner-Lehmkuhl ${ }^{5}$.

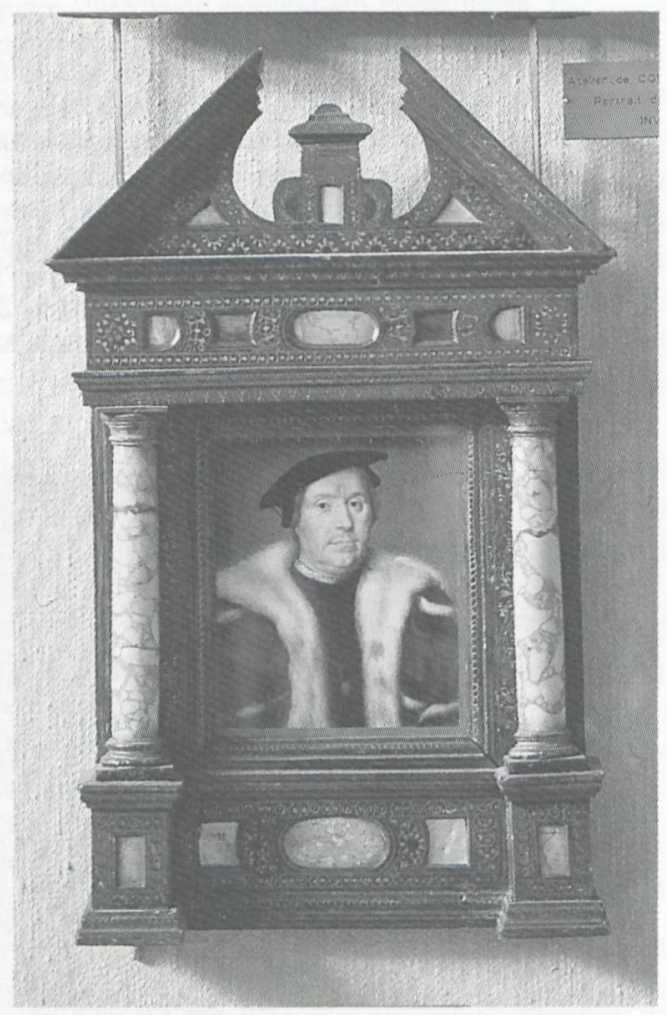

3. Corneille de Lyon, cadre architectonique d'un portrait de cour, Musée du Louvre.

Tout ce que nous venons de citer - et aussi le fait spectaculaire que l'auteur du cadre de la Vierge aux rochers avait reçu plus d'argent que Léonard de Vinci - se rapportent à une exécution parfaite des corps de retable (Altargehäuse), aux travaux de menuiserie (Kistlerei) concernant la production et la préparation des panneaux destinés à la peinture ains que leur montage dans un cadre orné de sculptures précieuses, puis doré. Parfois, les frais de dorure - à cette époque les feuilles d'or étaient encore très épais- ses - sont mis à part et absorbent la plus grande partie du coût total. Les fabricants de cadres ont des ateliers de menuiserie (Kistlerarbeit), de meubles, de sculpture et d'ornementation sur bois, de polychromie et de dorure. Le statut du maître qui dirige l'atelier, dont la signature nous est parvenue, ne découle pas de son activité de fabricant de cadres, mais de compétences très exigeantes. Au XVII siècle encore, la signature sur ces cadres n'est justifiée que par un savoir-faire et des capacités dépassant de beaucoup la simple fabrication des cadres : un Van der Meulen en Flandre, un Grinling Gibbons en Angleterre ou un Derk Daniels ${ }^{6}$ en Hollande étaient des sculpteurs sur bois réputés, qui ont exécuté des boiseries pour des palais et des églises ainsi que quantité de meubles. En Italie également, il existe au XVIII siècle des cas où un cadre, un siège ou une table ont été exécutés non pas par un menuisier, mais par un sculpteur.

Deux conclusions peuvent être tirées de ces considérations :

1. La fabrication de cadres pour les tableaux a surtout été reconnue comme un « art » au XVIII" siècle, à partir des ébénistes français ${ }^{7}$. Cette évolution coïncide avec les débuts de l'esthétique moderne, selon laquelle les œuvres d'art ont perdu leur lien direct avec la réalité ; elles s'adressent au goût, à l'intensité du sentiment.

2. Il existe une nette hiérarchie entre les travaux d'encadrement ambitieux, auxquels participent pour une part des sculpteurs en renom et l'objet moyen d'usage courant, qu'est un cadre de tableau. Au XVII' siècle déjà, des encadrements d'une valeur artistique remarquable ont été octroyés à des portraits et à des tableaux de batailles, mais l'encadrement restait avant tout limité aux peintures religieuses. Il existe une différence de rang manifeste entre les retables et leur réceptacle (Gehäuse) qui sont en rapport avec la liturgie, et les simples encadrements des tableaux de toutes sortes.

L'histoire des cadres ne peut donc être séparée de celle du changement de la fonction des tableaux ; liés auparavant à la liturgie et classés typologiquement, ils sont finalement devenus des « meubles ». En passant par les galeries des châteaux et les salles d'exposition des habitations aristocratiques, par un faste lié à la classe sociale, par les thèmes humanistes des peintures, les tableaux se sont diversifiés et sont devenus un vaste catalogue des traditions académiques. La création de galeries ouvertes au public coïncide avec l'époque des Lumières, mais c'est également le début de la liberté des thèmes, le bon plaisir dans ce que chacun accroche chez soi. Si on décrit ce processus de nivellement des fonctions en termes de genres artistiques, on constate jusqu'à la fin du Moyen Age la prédominance d'une liaison avec l'architecture, d'une phase pour ainsi dire « sculpturale », pendant laquelle les tableaux importants étaient entourés d'un cadre qui, par son répertoire de formes, mettait leur signification en relief. En conséquence, le dernier pas qui mène à détacher le tableau des rapports architectoniques, c'est la liberté de le placer selon son gré, ce qui ne nécessite plus que de délimiter le champ dans lequel il est perçu. L'instabilité des justifications esthétiques sur lesquelles se fondent les multiples conceptions de la peinture se manifeste clairement au XIX siècle dans leurs rapports avec le répertoire traditionnel des formes de cadres.

\section{La phase architectonique préparatoire}

Entre le XII et le XVI siècle, la richesse des types des triptyques est le fruit des usages et des prescriptions liturgiques. Lieu d'une vision peinte encadrée, les retables sont eux-mêmes une "église dans 
l'église ». C'est-à-dire qu'au même titre que l'édifice qui les entoure, ils incarnent un morceau du bâtiment du Salut, un passage typologiquement ordonné à travers l'histoire du Salut. Chacun connaît les suites de peintures en plusieurs parties dans lesquelles des indications sur le vieil Adam signalent le commencement des temps (ne serait-ce qu'au moyen d'un crâne figurant dans une Crucifixion), de même, sur le couronnement d'un retable, l'indication du retour du Christ signale la fin des temps (comme dans les retables sculptés sur bois des $\mathrm{XV}^{\mathrm{e}}$ et $\mathrm{XVI}^{\mathrm{e}}$ siècles). L'encadrement des panneaux des retables sculptés sur bois, les plus anciens, se rattache directement aux formes de la structure de l'église ; par la suite, les corps des retables adopteront une forme architectonique qui leur sera propre.

Si on examine la manière dont les tableaux sont disposés dans les retables, on distingue deux formes d'encadrement. La première, dispendieuse, consiste à créer une architecture destinée au retable, la seconde se contente d'un socle déterminé par des raisons constructives et d'un encadrement très simple pour les panneaux peints. Avec leur alignement rigide d'une série de peintures, bon nombre de retables italiens ne séparent pas toujours clairement ces deux façons de faire ; en revanche, elles sont nettement distinctes dans les retables à transformations du nord des Alpes.

$\mathrm{Si}$ on considère les encadrements postérieurs, destinés à des tableaux isolés, les différentes formes des cadres des retables apparaissent comme un stade intermédiaire, qui est un élément autonome d'une architecture chargée de signification. Cette architecture n'a pas été créée pour un tableau isolé afin d'expliquer ce qu'il représente, mais au contraire, le tableau inséré - ou la suite de tableaux - constitue une introduction visuelle au contenu déterminé par le corps du retable (Altargehäuse).

La préhistoire du retable a de multiples significations pour l'origine de l'encadrement du tableau autonome. Elle en détermine le contexte et présente le programme d'ensemble dont dépend le sujet de chaque tableau. On ne peut comprendre l'exigence didactique et la dignité des représentations figurées, et ce, jusqu'au cœur du XVIII' siècle, sans tenir compte de leur liaison spirituelle avec le monde des tableaux antérieur.

Le type canonique du cadre de tableaux suit une seconde ligne de tradition. Les superstructures architectoniques en forme d'édicules et de tabernacles ont des connotations significatives avec les portails d'églises, les retables et les tabernacles. C'est vrai des cadres de Clouet et de Corneille de Lyon destinés aux portraits des gens de la cour de France, c'est vrai aussi des cadres Sansovino pour les portraits des doges, jusqu'à la fin du XVIe siècle.

Pour finir, il faut encore signaler un troisième courant traditionnel : il s'agit des profils empruntés à l'architecture des portails et des fenêtres d'églises (vus de l'extérieur). Aux XVe et XVI $\mathrm{XV}^{\mathrm{e}}$ siècles, on trouve au nord des Alpes, dans les tableaux isolés des retables, comme dans les tableaux indépendants déjà " autonomes ", une gorge en forme d'ébrasement, issue du " larmier » destiné à éloigner les eaux de ruissellement. Le faux marbre de la polychromie d'une série de ces tableaux va dans le même sens. Il n'y a pas de contradiction à interpréter les profils et le faux marbre des encadrements anciens à partir des formes des plaques tombales ${ }^{8}$

L'ornementation des profils de cadres ne dépend pas entièrement de la tradition religieuse. C'est plutôt un emprunt direct aux modèles antiques qu'on peut observer dans l'architecture tant religieuse que civile avec les éléments architecturaux en saillie, tels que

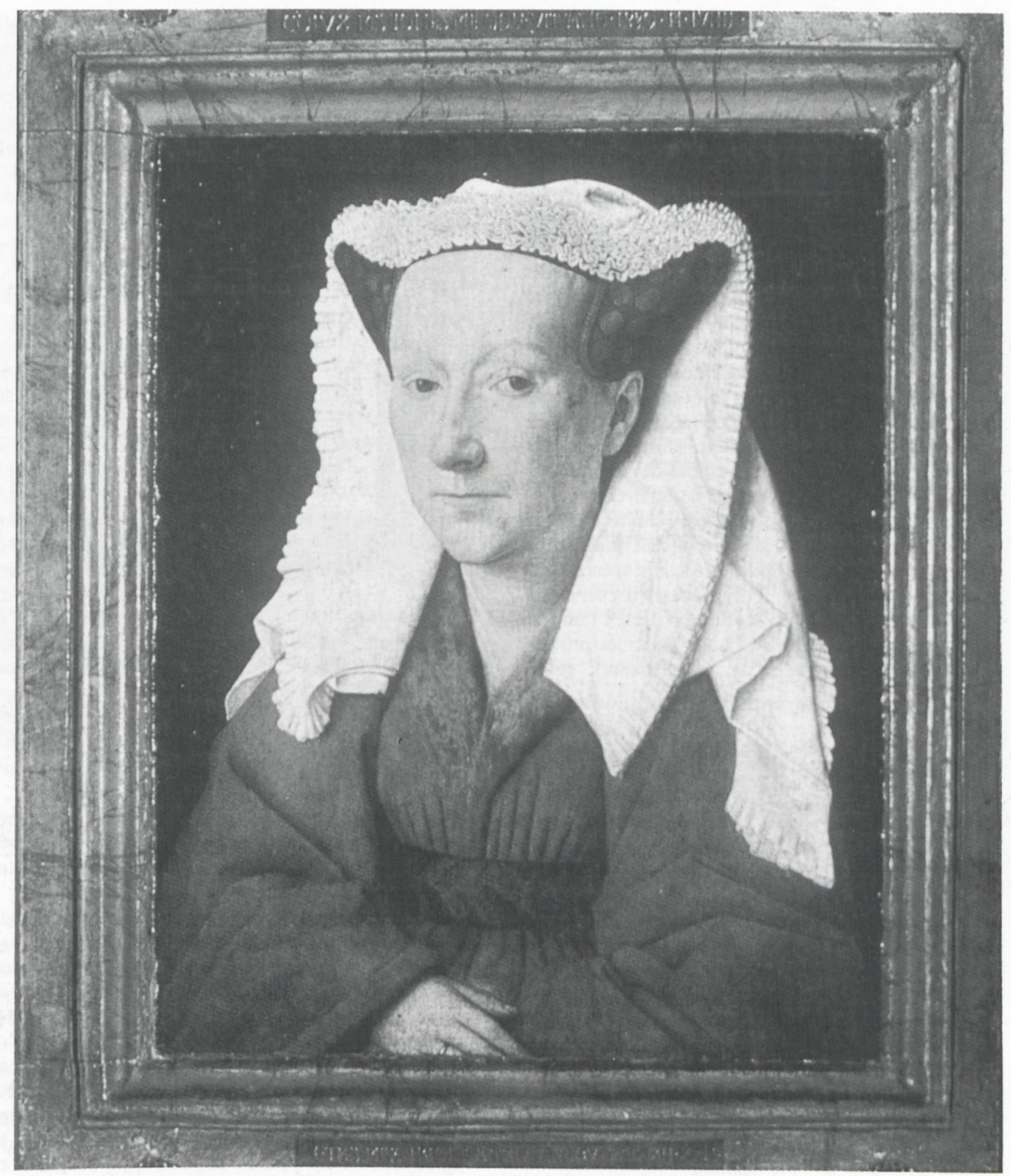

4. Face et revers d'un profil de cadre peint en faux marbre qui rappelle les représentations funéraires, Jan van Eyck, Portrait de Marguerite, épouse de l'artiste, 1439, Bruges, Groeninge Museum.

moulures, chapiteaux et linteaux de portes ; il y a également des emprunts au mobilier précieux, aux cassone italiens du Xve siècle, par exemple, qui présentent à la perfection les différents ornements des profils antiques. Ce répertoire classique sera repris sans cesse jusqu'au $\mathrm{XX}^{\mathrm{e}}$ siècle.

\section{Vers le tableau autonome}

Beaucoup sont surpris d'apprendre que les tableaux pareils à des objets déplaçables, qui ornent nos appartements, sont un phénomène culturel extrêmement récent, inimaginable jusqu'à la fin du Xve siècle. A notre époque où existent de nombreux musées de plein air et une vaste documentation sur l'histoire des sociétés ${ }^{9}$, on peut acquérir une vue approfondie des conditions historiques de l'habitation humaine. C'est seulement depuis le XIX' siècle qu'on trouve dans de larges couches de la bourgeoisie des salons et des cabinets de travail ornés de tableaux.

Dans les maisons paysannes, les logements d'ouvriers et de petits employés la décoration d'ima- ges est restée littéralement « médiévale » jusqu’à la fin du $\mathrm{XIX}^{\mathrm{e}}$ siècle. Les pièces étroites servant à la fois de salle à manger, de chambre à coucher, de cabinet de toilette, de salon et d'atelier ne laissaient guère d'espace libre pour accrocher des tableaux. La présentation de tableaux encadrés est le privilège des couches sociales supérieures ; la satisfaction des besoins vitaux primordiaux ne leur posant aucun problème, elles pensent à la culture et à l'édification et déjà un peu à la représentation sociale grâce à cette sorte de carte d'identité culturelle. Sans exclure un coin dédié à la dévotion avec des représentations figurées (crucifix) ou de petites images simplement posées (gravures coloriées, lithographies).

On objecte parfois qu'au XVII' siècle, aux PaysBas, les paysans et les petits artisans achetaient déjà des peintures. On peut alors se demander si ces faits peuvent être généralisés et être autre chose qu'insolites et exceptionnels, et s'il s'agit plus d'un investissement singulier que d'un désir effectif de meubler l'habitation. Nous connaissons bien ces ameublements grâce aux inventaires de faillite ou après décès. Nous connaissons aussi bon nombre de demeures historiques et 
le statut social de leurs habitants, et si les représentations d'intérieurs montrent que les murs recevaient plusieurs tableaux, dont certains de grande valeur à en juger par la beauté de leurs cadres, cela arrive exclusivement dans les couches les plus riches de la société, ce que confirme d'ailleurs le reste des inventaires.

Les peintures d'intérieurs qu'on rencontre de plus en plus souvent dans beaucoup de régions d'Europe depuis le XVII' siècle, apportent donc un précieux témoignage à l'histoire de l'encadrement. Mais leur valeur d'information sur les conditions effectives de l'habit dépend d'une interprétation rigoureuse. La plupart du temps, la représentation des intérieurs de la noblesse ou de la grande bourgeoisie est rehaussée d'allégories et stylisée. Ce sont pour ainsi dire des espaces "symboliques » dans lesquels est représenté un événement moralisateur. Les pièces d'habitation deviennent des scènes de théâtre illustrées par des séries de tableaux. Les représentations de cuisines sont une typologie - pour ne pas dire une encyclopédie de la cuisine et de ses ustensiles. Néanmoins, ces formes esthétiques liées à l'histoire recoupent quelquefois notre préoccupation des milieux sociaux spécifiques. Pour autant que je sache, il n'y a guère eu à cet égard que deux périodes avant la fin du XIXe siècle : l'une au milieu du XVII siècle en Hollande (avec les œuvres d'Adriaen van Ostade et de Brekelenkam), et en

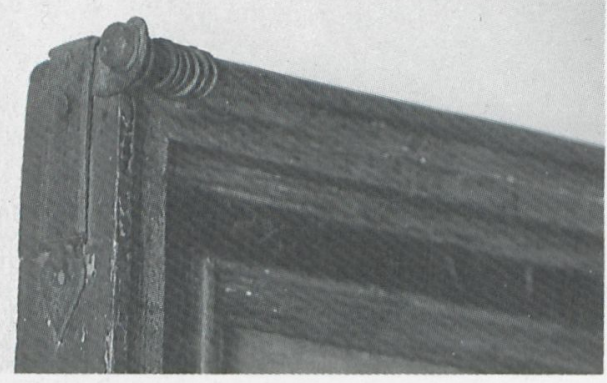

5. Cadre espagnol du XVI e siècle, avec sa tringle à rideau d'origine, Winterthur, collection Reinhart.

France au milieu et dans la seconde moitié du XVIII siècle (avec Chardin et Lépicié) et en Angleterre (Hogarth et Willkie).

Les choses peuvent se résumer ainsi. A partir du $\mathrm{XV}$ siècle, les représentations issues de la liturgie religieuse se détachent progressivement de celle-ci : petits retables de voyage, retables et tableaux destinés à des pièces consacrées à la dévotion privée, diptyques, tableaux de dévotion et portraits de donateurs, ainsi que des portraits de saints pour des fins de dévotion privée. Il faut se représenter ces tableaux principalement comme des objets posés sur un meuble, parfois conservés dans des réceptacles spéciaux, ou occasionnellement accrochés. Ils sont présentés dans un coin de pièce consacré à la dévotion, ce ne sont pas des objets d'ameublement.

Depuis la fin du Xve siècle, les tableaux, en premier lieu les portraits, font partie du mobilier artistique. Les aménagements les plus riches en tableaux se trouvent dans les salles d'apparat des châteaux et des palais. Dans les cabinets de curiosités spécialement aménagés on accroche les tableaux d'histoire, les allégories et les portraits, et de même dans les couloirs de communication ou "galeries", relativement bien éclairés des châteaux ${ }^{10}$.

A la fin du XVI siècle, en Italie et aux Pays-Bas une production indépendante de la commande appa-

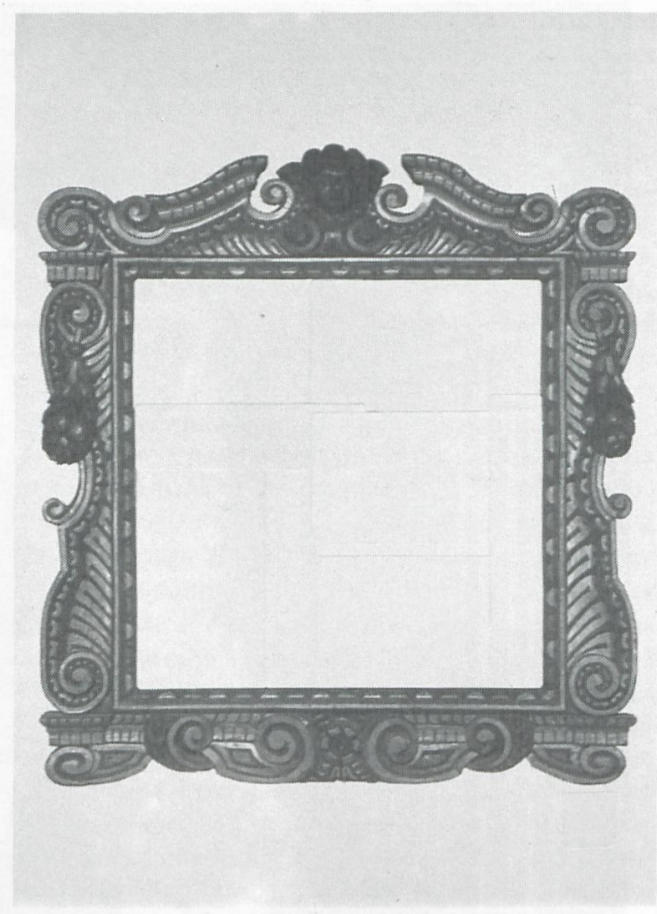

6. Combinaison des conceptions architectonique et sculp turale, cadre Sansovino, fin du XVIe siècle, Londres, Victoria and Albert Museum.

raît expressément réservée à un large marché d'acheteurs. Les tableaux sont pour la première fois encadrés en série. Ils sont vendus dans les foires, les ateliers, les magasins des commerçants et chez les libraires.

Les tableaux à sujets séculiers restent liés par leurs thèmes à des contenus historiques, religieux et philosophiques, ils conservent encore des fonctions d'enseignement moral, ils concrétisent l'érudition et les prétentions réthoriques pour les nobles et la riche bourgeoisie des villes, qui les achètent.

Pendant les XVII' et XVIII' siècles, à côté des thèmes de la commande traditionnelle, on voit des sujets de contenu plus pauvres : tableaux de mœurs, natures mortes, paysages. Les cadres mettent en valeur la signification esthétique ou philosophique propre à ces tableaux, leur format et leur facture suivent des normes générales avec des profils de moulures et une ornementation fidèles à quelques modèles de base communs à toute l'Europe.

Les tableaux ne seraient-ils qu'une forme passagère de l'objectivation du savoir liée au prestige social et au besoin de légitimation propres aux acteurs de quelques périodes de transition au cours desquelles les structures sociales se différencient? La question se pose, quand on observe la façon dont sont meublés les appartements modernes, pauvres en tableaux, surtout en cadres, et quand on considère, d'autre part, les représentations idéales des intérieurs artistocratiques que l'on trouve par exemple dans certaines Annonciations de la fin du Moyen Age : les images y sont utilisées d'une manière qui reste étrangère à l'histoire du tableau avec cadre ; dans les deux cas suffit un simple morceau de papier avec un dessin, une gravure, collé ou cloué au mur, ou même, de nos jours, une reproduction. L'Annonciation du Maître de Flémalle (vers 1430) au musée de Bruxelles représente ce que l'on considérait au Moyen Age comme mobilier précieux : des corniches, des bancs, des chandeliers et des pots artistement sculptés ou fondus. On y voit aussi une image, mais elle n'est pas encore traitée comme un meuble, elle n'a pas de cadre, elle est sans rapport durable avec la chambre où elle est simplement collée sur la cheminée de marbre avec un peu de cire à cacheter. Le papier endommagé montre qu'il ne s'agit que d'un supplément momentané et non d'un élément du mobilier.

\section{La phase « sculpturale 》}

La formule « architectonique » donne au cadre un caractère permanent, en quelque sorte comme la façade d'un édifice, un portail, un reliquaire ou une armoire. Cette forme figée, toute de dignité, est relayée à partir du Xve siècle, selon un principe différent, par un cadre qui entoure le tableau. Il le détache du mur environnant au moyen de quelques éléments architectoniques, moulures profilées, bandes ornées. Ces types de cadres mobiles et autonomes se sont formés en partie parallèlement au cadre architectonique, et en partie en élaborant des formes mixtes avec ce dernier. Le cadre, devenu logiquement la limite extérieure du tableau, se développe à partir du socle et de la bordure.

L'encadrement circulaire des tondi, et des petits formats présente des formes richement ouvragées, encerclant le tableau. Chez les Siennois, les Florentins et les Vénitiens du XVe siècle l'art de l'encadrement suit une évolution originale.

La fonction des retables étant étroitement associée à un lieu donné, on comprend parfaitement que ceux-ci aient avant tout conservé leur encadrement architectonique jusqu'au cœur du XvIII siècle. Jusqu'à la même époque, les peintures de grandes dimensions faites sur commande, destinées aux châteaux de la noblesse et aux palais, conservent dans leurs encadrements les formes fondamentales des édicules à niche. Et dans les ordonnances d'architecture intérieures on trouve des niches d'encadrement incorporées dans la construction. C'est dans cet esprit qu'il faut interpréter le dessin de Rembrandt, de 1656, pour La Leçon d'Anatomie du Dr. Deymann.

Durant tout le XVI siècle, ces deux formes se retrouvent l'une à côté de l'autre dans les miniatures, les dessins, les gravures, parfois même associées. La forme stricte des édicules à niches peut éclater en un décor qui tourne autour du tableau. L'évolution des cadres vénitiens dits cadres "Sansovino " offre des exemples parlants de cette réinterprétation. Les cadres « Lutma » hollandais, du XVII ${ }^{e}$ siècle, suivent la tradition vénitienne des décors de cuirs (Ohrmuschel- und Knorpelwerk) ; ils comportent un cadre plat (Plattenrahmen) bordant la surface peinte sur une largeur uniforme, ou éventuellement une surface plate à laquelle des bouquets et des trophées verticaux sont suspendus. Ce principe semble tout à fait se contredire lorsque de grosses têtes de putti rayonnant de l'intérieur vers l'extérieur sont disposés dans les cartouches d'angle, en soulignant le principe du cadre courant autour du tableau. On trouve cette même contradiction entre des moulures profilées «dormantes» entourant le tableau et un décor suspendu dans les cadres coûteux de Bologne, mais sous une forme d'où les disparates ont été prudemment éliminées. Les rinceaux des cadres provenant de l'atelier de Formigine et de ses successeurs sont à première vue ambigus, ils paraissent courir régulièrement autour du cadre, mais en examinant de plus près, on constate une orientation vers le haut et vers le bas. Le principe architectonique est repris de nouveau à la fin du XVII ${ }^{e}$ siècle, sous une forme transposée, dans les cadres à feuillages d'une richesse exagérée, vraisemblablement destinés surtout aux sujets religieux.

Les types dont nous venons de parler ne sont 
finalement qu'une minorité de plus en plus réduite dans la production européenne : des réalisations spéciales et coûteuses déployant tout un luxe de figures et

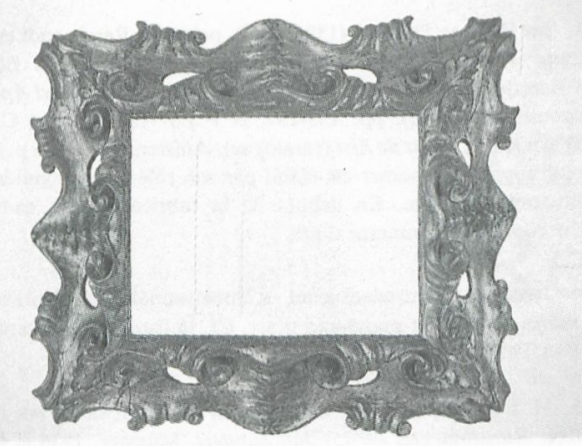

7. Cadre florentin du XVIIe siècle dérivant du type de cadre plat, mettant en évidence le caractère centrifuge de l'ornementation sculptée, Munich, F. Riggauer.

de décors sculptés. La majeure partie des cadres fabriqués aux $\mathrm{XVI}^{\mathrm{e}}$ et $\mathrm{XVII^{ \circ }}$ siècles est constituée par les cadres plats (Plattenrahmen) et les cadres moulurés dont l'évolution est caractérisée par la vigueur croissante du profil des moulures et par l'espace nettement incliné qui sépare le profil extérieur du profil intérieur des Plattenrahmen. Dans l'ensemble, les cadres deviennent plus larges, leur composition est plus dynamique. A celle-ci correspondent aussi la matière ou la polychromie de la surface : aux Pays-Bas, le luisant velouté des placages de bois précieux prend la relève des bois teintés, donc mats. Dans l'Italie, l'Espagne et la France du XVII siècle les cadres argentés et dorés remplacent les types antérieurs plus simples aux profils et à l'ornementation dorée sur fond noir.

Entre 1600 et 1650 , les tableaux des galeries donnent un certain aperçu de ce qu'on pouvait faire alors en matière d'encadrement. Ainsi qu'en témoigne le peu de cadres qui nous sont parvenus, le début du XVII siècle est dominé par la sobriété. Avec le renforcement des profils des moulures et des ornements sculptés, différentes traditions se constituent en Europe. Les cadres les plus puissants, les plus gigantesques par rapport à la surface peinte, se trouvent finalement à la fin du XVII* siècle et au début du XVII'* siècle.

Quand change la manière dont le spectateur apprécie la réalité du tableau, de multiples transitions artistiques s'imposent dans la prodigalité. On en comprend la nécessité en comparant par exemple les fresques de Michel-Ange à la Chapelle Sixtine avec celles de Carrache au palais Farnèse, postérieures d'à peine quatre-vingt-dix ans. Avec Michel-Ange, la peinture crée des figures et des échappées spatiales entre les doubleaux et les pilastres de l'architecture feinte. En revanche, chez Carrache, les tableaux mythologiques sont intégrés dans des encadrements emboîtés et multiples qui séparent des niveaux différents de réalité, en faisant même alterner des grisailles imitant la sculpture et des figures au premier plan traitées dans des couleurs franchement illusionnistes. Les conceptions esthétiques et les théories sont au XVII siècle une affaire académique et il n'est pas possible de dire dans quelle mesure elles ont pu conditionner un sentiment général. Pourtant, un certain accord avec les vues traditionnelles sur la réalité de l'image ${ }^{11}$ doit permettre de dire que la représentation artistique n'est jamais une copie ; elle est un langage acheminant vers une connaissance plus ou moins symbolique du réel. Par conséquent, pour les cadres hollandais il suffit de délimiter par rapport à l'observation banale une vision du réel approfondie de manière exemplaire, tandis que d'autres écoles exigeraient une réflexion plus large. $\mathrm{La}$ contribution de la peinture peut aussi résider dans un dépassement de la perception personnelle, une fusion poétique de ce monde avec des formes et des caractères esthétiques du passé, ou bien dans une élévation à l'extase, dans une réfraction théâtrale de la réalité. Dès lors un rapport peut exister entre le classicisme poétique de la peinture française du XVII siècle et le luxe des cadres Louis XIII avec leurs feuilles d'acanthes étirées et, un peu plus tard, avec les cadres Louis XIV couverts d'ornements, qui se sont développés à partir de la cimaise.

Cette esquisse sommaire appellerait un complément d'explication, ne serait-ce qu'en rappelant que leur emploi est limité par l'intervention d'autres types de cadres comme les séries de profils strictement inexpressifs dans le genre des cadre "Salvatore Rosa » ou «Maratta ». Si l'on examine les tableaux d'intérieurs de l'époque de Vermeer, on constate, comme en Angleterre et en France, que plusieurs traditions formelles s'y côtoient. Des cadres Maratta classiques, on passe par les formes Louis XIII pour arriver aux cadres « Lutma » (ou « Sunderland ») et aux sombres cadres en ébène.

Cette période « sculpturale » est en gros celle où les formes des cadres sont les plus diversifiées, la sculpture la plus abondante, les applications et les incrustations les plus variées : métal, pierres dures, nacre, ivoire, dents de baleine, etc., celle aussi, où on utilise une grande variété de techniques de gravure, de ciselure et de dorure.

\section{Le XVIII siècle}

Si on laisse de côté un instant la multiplicité des formes ornementales pour considérer uniquement les profils des moulures, on s'aperçoit que la richesse des formes et l'ampleur de la composition se perdent au plus tard sous la Régence. C'est alors que les moulures s'aplatissent et se simplifient ; les gorges rondes se transforment en ondulations molles, et les successions de profils, auparavant séparées, concourent à l'impression générale. Le profil extérieur devient une large bande qui domine sur le plan optique se termine simplement du côté du tableau par de fines raies inter-

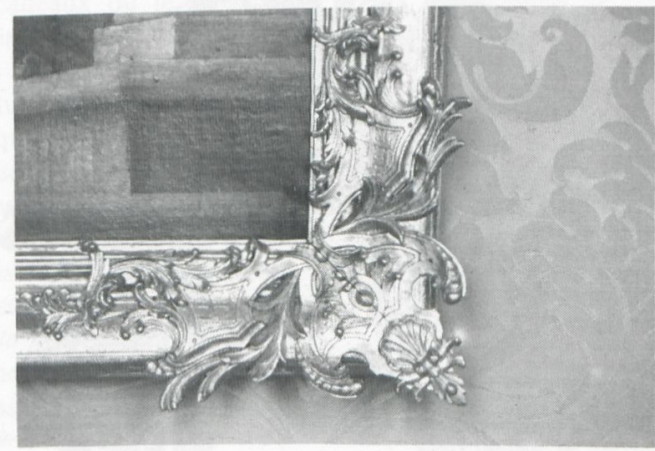

8. Munich, Résidenz, Grüne Galerie, 1735-1740, Opposition entre le profil de cadre aplani et l'ornementation plate, dans la transition entre les styles Régence et Louis $X V$.

médiaires, joue aussi un rôle dans cette impression d'ensemble. L'ornementation Régence et Louis XV ne se contente pas d'organiser la surface, elle «surmonte » le profil sans l'abolir. C'est ce qui fait en particulier le charme, des cadres sculptés Louis XV, où dans les angles l'ornementation se libère largement des traits de la construction sans la détruire. L'ornementation délicate des cadres anglais, effilochée sur les côtés, représente une forme spéciale, de même que les cadres rococo allemands brisés aux angles d'où prolifèrent des languettes et des oreilles (Zungen und Ohren) dans des variations d'une fantaisie extraordinaire.

Sous la Régence, déjà, le graphisme de l'ornementation domine l'impression générale; les motifs jouent au-dessus des surfaces limitées par les profils. Dans la décoration Louis XV, les moulures ne se retrouvent que dans les angles, leur cours est partiellement subordonné aux courbes et oscillations des champs d'ornements. Le caractère sculptural des profils s'élevant vers l'extérieur est réinterprété par les structures ornementales, tandis que les moulures qui, autrefois créaient la profondeur, s'atrophient et s'aplatissent. Après le milieu du siècle, dans les cadres de «transition » ou Louis XVI tout effet de profondeur est abandonné et l'ornementation se réduit à des motifs discrets au relief insignifiant. Les cadres Louis XVI sont caractérisés en particulier par des motifs sculptés qui prennent un même caractère de fleurs et de bouquets serrés.

\section{Recombinaisons « pittoresques »}

Un saut quantitatif s'est produit depuis la fin du XVIII siècle dans la littérature européenne en raison de l'arrivée d'un large public de nouveaux lecteurs; de même, les tableaux et les cadres ont été conçus d'une manière toute nouvelle, puisqu'il ne s'agissait plus de satisfaire les commandes raffinées des aristocrates. On le constate en observant que de nombreux cadres sont signés dans la période antérieure à la Révolution Française, et qu'il y a fort peu de signatures après celle-ci. Non pas que les fabricants de cadres aient eu alors une moindre conscience de leur valeur, mais simplement parce qu'on ne donnait plus à ces artisans d'élite qu'un très petit nombre de cadres à sculpter. Les cadres du XIXe siècle sont presque exclusivement une marchandise coupée et collée vendue au mètre. L'ornementation est faite de diverses matières malléables appliquées sur des bois profilés; les cartouches sont rapportés après collage sur les assemblages des angles. Depuis l'Empire, les cadres n'ont même plus cette ornementation délicate couvrant la surface ; ils se composent principalement d'une gorge qui donne l'impression assez fâcheuse d'être lisse et nue. Cet aspect lisse résulte souvent du caractère métallique de la dorure (Fassungscharakter). Dans la mesure où les ornements sont rapportés, ils relèvent d'un schématisme rigide, avec des feuilles d'acanthes introduites avec toute leur raideur et l'étrangeté d'un motif historique. La gorge, élément dominant, est limitée du côté du tableau par un jeu de moulures fines, et du côté extérieur par une arête vive, avec ou sans profils. Ce schéma de base peut être varié par des redents et par l'extension de la région profilée, mais il est traité de telle sorte que la disposition géométrique fondamentale du cadre et la trame du décor rigoureusement stylisé conservent leur force esthétique propre arbitraire autour de la peinture.

La diversité des encadrements réalisés au XIX`e et au début du XXe siècle ne permet pas de les ramener à un petit nombre de concepts directeurs. Les projets de cadres sculptés et moulés qu'Ingres a conçus, ou les encadrements réalisés d'après les dessins des préraphaélites anglais, mais aussi les performances, couronnées en leur temps, des écoles éclectiques, principa- 
lement en Italie, sont aussi des créations artisanales de haut niveau. Cependant, si l'on se réfère à la typologie générale des profils, cela ne va pas sans quelques caractères communs au sein de cette variété. Le milieu et la seconde moitié du XIX' siècle sont marqués par des liaisons de plus en plus diversifiées avec les modèles des styles antérieurs : chez les préraphaélites, ce retour se traduit par des zones de décoration richement ornées et parfaitement plates, sur des cadres très larges; pour les formes " néo-baroques », il peut s'agir d'ornements et de profils en plâtre imposants, massivement entassés ; pour les cadres dans l'esprit « vieil allemand » ou " vieil hollandais » il peut s'agir de Flammleistenrahmen noirs à rédents, le plus souvent en pente vers l'extérieur et largement débordants. Cependant, la plupart du temps ces types différents ont en commun un bord intérieur clair ou foncé s'étendant en partie comme une manchette et s'éloignent de la surface du tableau proprement dit. Bon nombre de «cadres impressionnistes » utilisent le même principe qui réside ici dans l'adaptation d'un cadre original ancien déjà patiné ou négligemment enduit, qui prend un caractère étranger par l'introduction d'une bande intermédiaire.

Le « cadre impressionniste » démontre aussi la fin de la fonction originelle du cadre. Cette fonction était en quelque sorte celle d'une bordure, d'une enveloppe, d'une mise à distance significative. Cet élément intermédiaire entre un environnement banal et la sphère particulière de perception qu'est le tableau se traduisait par l'architecture générale, les structures architectoniques et l'ornementation du cadre. Depuis le XIX' siècle, ces traits ont perdu le sens qui les unissait en faveur de constructions esthétiques originales. Celles-ci ne provoquent plus qu'une impression d'ensemble, artistique ou historique, en manifestant simplement un décor subordonné au goût et à l'impression que l'encadreur éprouve devant chaque tableau.

Passé ce seuil, il existe certes toujours des formes typiques : citations, réalisations pittoresques et ornementales dotées d'un grand charme, mais le caractère

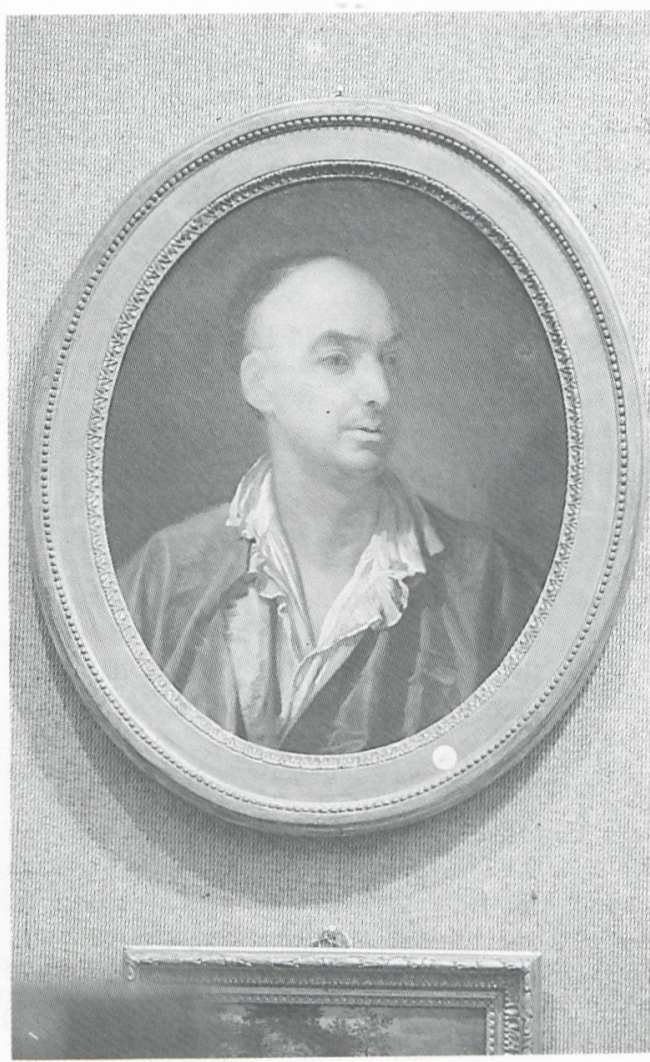

9. Portrait de Diderot, par J.B. Greuze, dans son cadre original signé " H. Letonné » (reçu maître en 1773), Londres, Christie's, 1985. Les encadreurs ont commencé à signer leurs œuvres après l'épanouissement du genre.

de plus en plus subjectif du tableau ne permet plus de développer une caractéristique bien frappée des encadrements. Le sujet autonome ne se soumet à aucun cadre.

Traduit de l'allemand par Jacques Chavy
NOTES

1. W. Ehlich, voir bibliographie, p. 60-61

2. Diderot et d'Alembert, Encyclopédie, Paris, 1751-1772.

Sur Herman Doomer (1595-1650), peint par Rembrandt en 1640 (New York, Metropolitan Museum), voir: I.H. van Eeghen, " Baertjen Martens en Herman Doomer ", in Maandblad Amstelodamum, 43 (1956), pp. 133-137, et P.J.J. van Thiel et C.J. de Bruyn Kops, Prijst de lijst (catalogue), Amsterdam, 1984, p. 31. Le rang social de Doomer est établi par son rôle dans la guilde et la situation familiale. En dehors de la fabrication des cadres, il s'occupait du commerce d'art.

4. Isaak van Ruysdael était «Ebbelystmaker » (fabricant de cadres en bois) et marchand d'art. Cf. la liste des fabricants chez Van Thiel, op. cit., note 3 .

5. H. Lerner-Lehmkuhl, Zur Struktur und Geschichte des Florentiner Kunstmarktes im 15. Jahrhundert, Münster, 1936 et Gilbert C., Revue de l'Art, n 37, 1977, p. 9.

6. D. Green, Grinling Gibbons, Londres, 1964 ; B Dubbe, "De Deventer beeldhouwer Derck Daniels (1632-1710) », in Antiek, 16, 1982, pp. 361-362, et Van Thiel, op. cit., note 3.

7. Comte de Salverte, Les ébénistes du XVIII siècle, Paris, 1962 ; F.L. Heckley, $A$ directory of antique French furniture, 1735.

8. M. Cämmerer-George, voir bibliographie, p. 62.

9. Cf. Indications bibliographiques dans G. Benker, Bürgerliches Wohnen, Munich, 1984, de même dans K. Kühnel (éd.), Alltag im Spätmittelalter, Graz/Vienne/Cologne, 1984.

10. Cf. Exemples pour la fin du xvı siècle dans N. Lieb, Die Fugger und die Kunst im Zeitalter der Spätgothik und der frühen Renaissance, Munich, 1952 ; N. Lieb, Octavianus Secundus Fugger und die Kunst, Tübingen, 1980.

11. Voir les traités concernant l'« inventio ", la « maniera " elle "concetto ". Cf. J. Shearman, Mannerism, style and civilisation, Harmondsworht, 1967 ; D. Mahon, Studies in seicento art and theory, Londres, 1947. C. Nordenfalk, " Realism and idealism in the portraits of Queen Christiana ", in Studies in Renaissance and Baroque Art presented to Anthony Blunt, Londres, 1967, pp. 122 sqq.

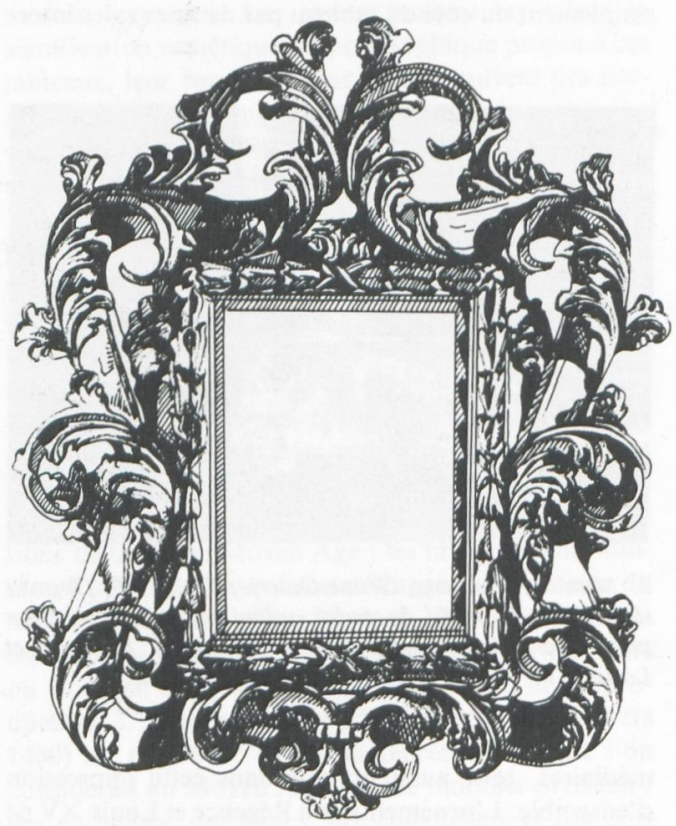

